\title{
Croissance des taillis de châtaignier en France : utilisation des données de l'Inventaire forestier national
}

\author{
M Bédéneau \\ INRA, unité expérimentale biomasse forestière et forêt paysanne, F 45160 Ardon, France
}

(Reçu le 26 janvier 1993; accepté le 30 septembre 1993)

\begin{abstract}
Résumé - On étudie l'évolution, en fonction de l'âge, de la biomasse ligneuse sèche sur pied dans le cas du châtaignier traité en taillis, à partir des données de l'Inventaire forestier national. Pour obtenir des courbes de croissance en biomasse et en hauteur, dans le cas du taillis pur, on retient 374 points d'échantillonnage, répartis sur 9 régions forestières, représentant $35 \%$ des points de sondages IFN. Un tarif permettant de déduire la masse totale du brin, en fonction de la circonférence à 1,30 m est appliqué à tous les brins des points de sondage retenus. On se sert de la mesure de l'accroissement radial à $1,30 \mathrm{~m}$ pour reconstituer, à l'aide du même tarif, la biomasse présente il y a 5 ans. On étudie la répartition de ces données à l'aide d'une analyse factorielle des correspondances, puis on ajuste des courbes d'évolution en biomasse et en hauteur, selon un modèle logistique généralisé. On obtient : hauteur dominante $(e n)=13,8 \times[1-\exp (-0,130 \times$ âge $)]$; biomasse $\left(e n t \bullet\right.$ ha $\left.{ }^{-1}\right)=67 \times[1-\exp (-0.19 \times$ âge)] $]^{6,09}$. Le maximum de production courante, pour le modèle moyen, est de 4,3 to ha- $\bullet^{-1}$ an $^{-1}$ à 9 ans, la production moyenne maximale est de 3 toha $\mathbf{a}^{-1} \cdot \mathrm{an}^{-1}$ à 12 ans. Ces chiffres sont inférieurs à ceux proposés dans une précédente publication. On montre que les régions forestières ont des potentialités de croissance différentes, mais que les modèles proposés peuvent toutefois en rendre compte. Des hypothèses sont avancées pour expliquer ces divergences. Des voies de recherches sont proposées.
\end{abstract}

taillis / biomasse / accroissement / modèle mathématique / inventaire forestier / Castanea

Summary - Growth of chesnut coppice in France based on IFN data. The total standing woody biomass of sweet chesnut coppice is studied using IFN data (French National Forest Survey). To obtain pure coppiced standing, 374 sample plots are selected on 9 forest regions, representing $35 \%$ of measured sweet chesnut plots. The equation giving total above-ground biomass of individual trees in relation to their girth at breast height is applied to the plots. Using the radial increment of $5 \mathrm{yr}$, the biomass present 5 yr ago is computed with the same equation. We consider data structure, and a general logistic model is applied to estimate height and biomass growth. The equations obtained are: dominant height $(m)=13.8 \times[1-\exp (-0.130 \times$ age $)]$ biomass $\left(t^{-h} a^{-1}\right)=67 \times[1-\exp (-0.19 \times \text { age })]^{6.09}$. The mean biomass growth model gives a maximum current annual increment of 4.3 toha-10yr-1 at $9 \mathrm{yr}$, 
and a maximum mean annual increment of 3 toha-1 $y r^{-1}$ at $12 \mathrm{yr}$. The results below are those presented in a previous paper. We show that forest regions have different growth potentials, but the proposed models can explain these differences. Future research work using the same data are proposed.

coppice / biomass / increment / simulation / inventory / Castanea

\section{INTRODUCTION}

Les études sur les potentialités de production de biomasse ligneuse des peuplements forestiers ont, d'après Bouchon (1974), 2 origines:

- le prix du matériau que se procurent les industries du bois est davantage lié à son poids qu'à son volume, tout du moins pour le bois d'industrie ;

- le fonctionnement d'un arbre, d'un peuplement forestier est mieux expliqué par la masse de matière produite que par son volume.

De nombreux auteurs ont publié des tables sur la biomasse des peuplements forestiers (Pardé, 1980 ; Cannell, 1982). II ressort de ces études que le régime du taillis est particulièrement bien adapté à la production rapide de biomasse ligneuse. Une préoccupation plus récente, l'intensification de la production ligneuse à vocation énergétique, a donné lieu à de nouvelles études :

- inventaire des potentialités de production de biomasse. On peut citer Lavoie et Vallée (1982) au Canada, Paavilainen (1981) en Finlande, Cost et McClure (1984) dans le sud des États-Unis, Auclair et Bigé (1984) dans la région Centre en France ;

- mise en place de nouveaux peuplements, utilisant des techniques de cultures intensives (irrigation, engrais, récolte mécanisée) et du matériel végétal sélectionné ; par exemple, peupliers en France (AFOCEL, 1983), eucalyptus au Brésil (Tomaselli, 1982), saules en Suède (Sennerby-Forsse et Mitchell, 1987).
Cette contribution s'inscrit dans le premier type de recherches : inventaire des potentialités des taillis en place. Le châtaignier (Castanea $s p$ ) est en effet une essence importante en France : il couvre $570000 \mathrm{~h}$, soit environ $4 \%$ de la surface boisée métropolitaine, c'est la troisième essence feuillue après les chênes et le hêtre, avec 81 millions de $\mathrm{m}^{3}$ sur pied. Les 2 tiers de cette ressource sont en taillis, $95 \%$ se trouvent en forêt privée. Il s'agit donc de préciser les potentialités de production des taillis de châtaignier, ceci dans 2 buts :

- détermination de la masse sur pied à un âge donné - dans un souci de gestion (exploitation de biomasse indifférenciée, conversion en futaie...);

- compréhension de la croissance des rejets de souches, et des divers modes de fonctionnements de cette essence selon le régime sylvicole.

Cet article fait suite à une première étude (Bédéneau, 1988) dans laquelle un modèle de croissance en biomasse du châtaignier traité en taillis simple avait été proposé. Ce modèle avait été établi à partir des mesures de placettes temporaires installées sur le territoire métropolitain par divers organismes de recherches ou de développement. Les écarts entre les points expérimentaux et le modèle théorique n'avaient pu être expliqués par les relations entre variables étudiées, hauteur, âge en biomasse. Outre des modes de fonctionnement différents, on a émis l'hypothèse d'un échantillonnage pouvant être biaisé.

Comme Auclair et Bigé (1984), les données utilisées sont celles de l'Inventaire 
forestier national. II s'agit ici des relevés de terrain des points de sondage, les données sont structurées par unité géographique ; la région forestière, "région naturelle formant une entité géographique, écologique et socio-économique» (IFN, 1985).

II s'agit donc à l'aide de ces données de vérifier si un modèle unique peut être proposé, et, si non, d'expliquer les variations de croissance entre régions.

Les chiffres de biomasse sont exprimés en tonne de matière sèche (masse ligneuse séchée à $105^{\circ} \mathrm{C}$ à l'étuve jusqu'à poids constant), il n'y a pas de distinction entre nécromasse et biomasse : il s'agit de «masse ligneuse totale sur pied".

\section{MATÉRIEL ET MÉTHODES}

\section{Données}

Bien que déjà décrites par ailleurs (Ottorini et Nys, 1981 ; IFN, 1985 ; Auclair et Bigé, 1984 ; Guéro et Houllier, 1990), nous faisons ici un bref rappel des méthodes de l'IFN.

L'unité d'échantillonnage au sol de l'IFN est composée de 3 placettes circulaires concentriques : $6 \mathrm{~m}, 9 \mathrm{~m}$ et $15 \mathrm{~m}$ de rayon. Elles correspondent - respectivement - à la catégorie des petits bois, moyens bois et gros bois, c'est-à-dire de diamètre mesuré à $1,30 \mathrm{~m}$ inférieur à 22,4 $\mathrm{cm}$, compris entre 22,5 et $37,4 \mathrm{~cm}$ et supérieur à $37,5 \mathrm{~cm}$. Le point d'échantillonnage est d'abord décrit : structure du peuplement, composition par essence, évolution, puis par essence, l'importance (pure, prépondérante...), la dimension (arbre de surface terrière moyenne), l'âge et l'importance de la régénération. Ensuite, sur chaque arbre, les caractéristiques suivantes sont mesurées : la circonférence à la base, le diamètre à $1,30 \mathrm{~m}$, à ce même niveau l'épaisseur d'écorce et la largeur des 5 dernières cernes d'accroissement, la hauteur totale, l'accroissement en hauteur des 5 dernières années. Diverses autres variables sont mesurées qui permettent d'établir un volume précis de l'arbre, telles, par exemple, le diamètre à $2,60 \mathrm{~m}$ et la hauteur de découpe de la bille de pied. Ces ren- seignements font objet d'un encodage propre à I'IFN, puis de traitements informatiques particuliers. L'IFN publie les résultats par département, sous forme de 2 tomes, un premier traitant des caractéristiques forestières et de l'étude des formations forestières de production, un second donnant le détail des formations boisées de production (nombre de tiges, volume, accroissement, etc) par types de peuplement, types de propriétaires...

\section{Mise en forme des données}

On a utilisé le fichier "arbre" du châtaignier prépondérant traité en taillis dans toute la France (prépondérant : dont le couvert libre est égal à $75 \%$ du peuplement considéré). Ce fichier représente 73395 arbres regroupés en 4525 points d'échantillonnage, répartis dans 181 régions forestières.

Les données ont été structurées en base de données. Pour đéterminer la masse d'un brin à partir de sa circonférence, nous avons appliqué à chaque brin le tarif suivant (Bédéneau, 1988): biomasse sèche du brin (en $\mathrm{kg})=0,184+$ $0,00296 \times C^{2,6}$, où $C$ est la circonférence à $1,30 \mathrm{~m}$ en $\mathrm{cm}$. La biomasse à l'hectare a été obtenue en tenant compte de la surface d'extension du point IFN.

Ce tarif «brin" n'étant valide que pour les brins vivants de châtaignier, on a ôté les points comportant des arbres morts. Du fait que l'on ne s'intéresse qu'au traitement en taillis simple, dans lequel aucune opération sylvicole n'a eu lieu, et où l'espèce est pure, on a éliminé les points où il y a eu passage d'éclaircies, où il y a présence d'arbres de futaie et où l'espèce est en mélange.

On n'a retenu que les points présentant un nombre suffisant de brins, la limite a été fixée à 1000 brins par hectare, ce qui correspond à une très basse densité (Rullier-Bréval, 1985).

Enfin, le nombre de points de sondage de I'IFN étant proportionnel à la surface occupée par l'espèce, on a retenu 9 régions forestières, représentant $35 \%$ des points d'échantillonnage. II s'agit des régions suivantes : "BassesCévennes", "Baugeois", "Châtaigneraie limousine", pays des Yvelines et de Fontainebleau (nommé "Région parisienne" dans la suite de cet article), "Périgord blanc", "Périgord noir", "Plateau limousin", «Plateaux et collines du BasDauphiné", "Terres rouges". On obtient finale- 


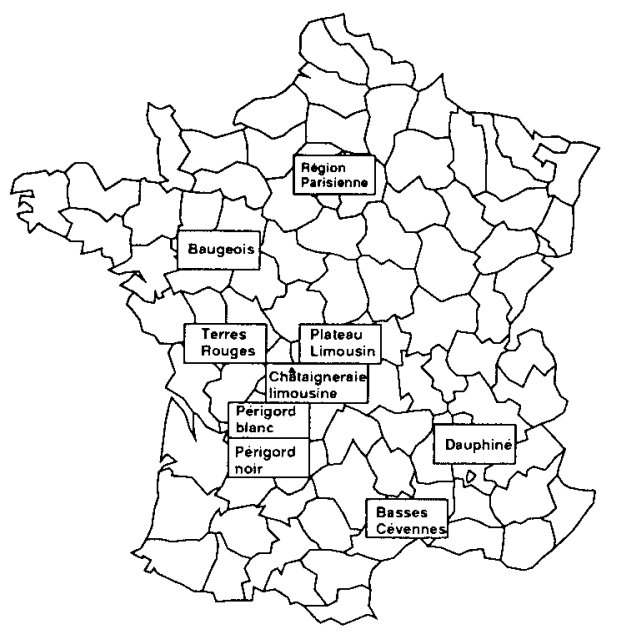

Fig 1. Situation des régions forestières étudiées.

ment un ensemble de 374 placettes de taillis pur.

L'âge de la placette a été déterminé comme la moyenne des âges des arbres sondés, plus ou moins 5 ans. Cette contrainte est imposée par le mode de mesure de l'IFN : lâge est déterminé à la souche sur 2 arbres, mais parfois sur plus, et peut être recodé en classe d'âge (Guéro et Houllier, 1990).

La hauteur, appelée hauteur dominante de la placette, a été obtenue en faisant la moyenne des 3 plus grands brins de la placette.

L'accroissement sur 5 ans est reconstitué à l'aide de la mesure de l'accroissement radial correspondant; on fait alors l'hypothèse que le nombre de brins est constant.

On a procédé de la même façon pour les hauteurs ; cependant dans certains cas l'accroissement en hauteur est nul, soit non mesuré ou mesurable, soit effectivement égal à zéro; on a alors considéré que l'accroissement en hauteur était nul.

\section{Méthode}

\section{Analyse factorielle des correspondances}

Le tableau initial est composé de 374 observations et de 5 variables : biomasse, âge, hauteur, accroissement en hauteur, accroissement en biomasse. Les observations sont repérées par leur numéro et leur région d'appartenance.

Les variables ont été chacune découpées en 4 classes d'effectifs égaux, puis éclatèes en variables logiques, 0 ou 1 . Les régions ont été recodées en 9 variables logiques. On a ensuite construit le tableau de contingence Région $\mathrm{X}$ Variables.

Sur ce tableau on a pratiqué une analyse factorielle de correspondances, afin de visualiser la structure des données. Du fait que l'accroissement en hauteur n'a jamais été mesuré dans la Région parisienne, on a fait une seconde analyse en portant cette région comme individu supplémentaire, ne participant pas à l'analyse.

Ces recodages et calculs ont été effectués à l'aide du logiciel ADDAD (1989).

\section{Ajustements}

Pour décrire l'évolution des peuplements on a employé le modèle logistique généralisé :

$$
Y=a \cdot\left[1-\exp (-k \cdot(t-t 0))^{1 / 1-m}\right.
$$

dans lequel $Y$ est la variable étudiée; $t$, l'âge; et a, $k$ et $m$, les paramètres à estimer.

Ce modèle a été retenu car il se montre efficace pour décrire des croissances en biomasse (Pages, 1986), et des croissances en hauteur (Osumi, 1983).

On a utilisé la technique de régression nonlinéaire (Tomassone et al, 1983). Les ajustements ont été effectués à l'aide du logiciel CS-NL (Bouvier et al, 1985). Le tableau étudié est celui des 374 points originaux, et des mêmes points affectés de hauteurs et biomasse 5 ans avant la mesure (soit un ensemble de 748 points).

\section{RÉSULTATS}

\section{Analyse des données}

Les 4 premiers facteurs principaux de l'analyse de correspondance rendent compte de $92,54 \%$ de la variance des données. La figure 2 montre les résultats obtenus avec la première analyse, sur les axes 1 et 2 , qui 
expliquent $71,62 \%$ de la variance. Les données sont liées entre elles : la production de biomasse et la croissance en hauteur sont fonction de l'âge. L'axe 1, déterminé par la croissance en biomasse, classe les régions par âge, et par productivité : les placettes âgées produisent peu, les jeunes ont un fort accroissement. Le long de l'axe 2 on retrouve une partition identique selon les croissances en hauteur ; les régions de peuplements âgés du côté négatif de l'axe, celles de peuplements plus jeunes vers le haut. La Région parisienne se trouve très loin sur l'axe 2 car les accroissements en hauteur n'y ont pas été mesurés, leur valeur est nulle ; en revanche sa hauteur est supérieure à $15 \mathrm{~m}$ et sa biomasse sur pied supérieure à 80 tha ; elle est donc proche de ces variables, avec les Terres rouges et le
Périgord noir. Comme l'accroissement en hauteur décroît avec l'âge, on trouve projetées sur le premier axe du côté négatif à la fois les placettes les plus âgées et les placettes dans lesquelles cette variable n'a pas été mesurée.

Les résultats de la seconde analyse (variance expliquée : $77,33 \%$ ) sont présentés en figure 3 . On retrouve l'axe 1 déterminé par la croissance en biomasse, l'axe 2 par la croissance en hauteur. Le bruit apporté par la Région parisienne disparaît : les variables de croissance en biomasse suivent les variables d'âge. Sur l'axe 2 , on retrouve cette partition avec les croissances en hauteur.

Dans les 2 analyses les hauteurs et biomasses observées sont assez peu discriminées par l'âge. Cependant dans la pre-

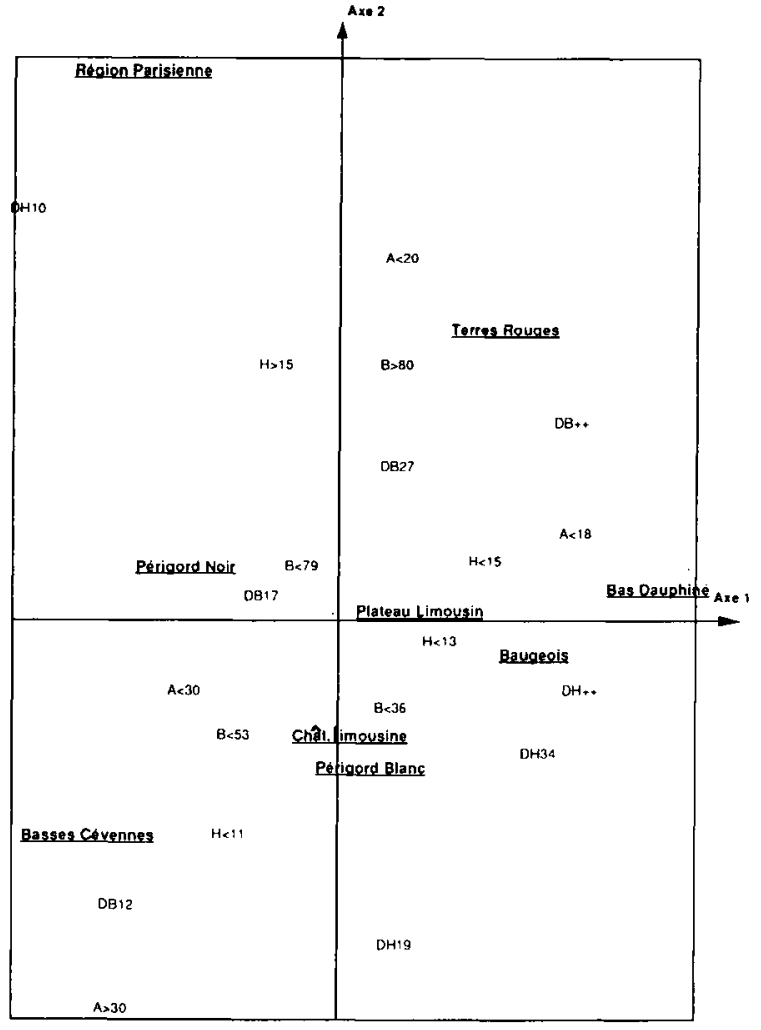

Fig 2. Analyse factorielle des correspondances du tableau de contingence de classes de variables dendrométriques par région. $A<18$ : âge <à 18 ans, $A<20$ : âge $<$ à 20 ans, $A<30$ : âge < à 30 ans, $A>30$ : âge > à 30 ans, $B<36$ : biomasse à I'hectare $<36 \mathrm{t}, \mathrm{B}<53$ : biomasse à l'hectare $<53 \mathrm{t}, \mathrm{B}<79$ : biomasse à l'hectare $<79 \mathrm{t}, \mathrm{B}>$ 80 : biomasse à l'hectare $>80 t$, $H<11$ : hauteur $<11 \mathrm{~m}, \mathrm{H}<13$ : $11 \mathrm{~m}<$ hauteur $<13 \mathrm{~m}, \mathrm{H}<15$ : $13 \mathrm{~m}<$ hauteur $<15 \mathrm{~m}, \mathrm{H}>15$ : hauteur $>15 \mathrm{~m}, \mathrm{DB} 12$ : accroissement en biomasse $<12$ tha, DB17 : 12 tha < accroissement en biomasse $<17$ tha, DB27 : 17 tha < accroissement en biomasse $<27$ tha, $\mathrm{DB}++$ : accroissement en biomasse $>27$ tha, DH10 : accroissement en hauteur $<10 \mathrm{dm}(\mathrm{Ou}=0)$, DH19 : $10 \mathrm{dm}<$ accroissement en hauteur < $19 \mathrm{dm}$, Dh34 : $19 \mathrm{dm}<$ accroissement en hauteur $<34 \mathrm{dm}, \mathrm{DH}++$ : accroissement en hauteur $>34 \mathrm{dm}$. 
mière analyse, sur le second axe, il y a variation simultanée de biomasse et hauteur. Ceci tendrait à dire que les placettes les plus grosses sont aussi les plus hautes, ce qui n'est pas très surprenant. C'est pourquoi les ajustements se feront sur les variables de biomasse et de hauteur.

\section{Ajustements}

Le modèle moyen de croissance en hauteur est le suivant :

$$
H_{0}=13,8 \cdot(1-\exp (-0,13 \bullet a ̂ g e))
$$

avec $\mathrm{H}_{0}=$ hauteur dominante (en $\mathrm{m}$ ). (Écart type résiduel : 2,77)
La croissance en biomasse est représentée par l'ajustement:

$$
B=67 \bullet(1-\exp (-0,192 \bullet a ̂ g e))^{6,09}
$$

avec $\mathrm{B}=$ biomasse à l'hectare (en tonnes). (Écart type résiduel : 35,26).

\section{Structure des données}

Sur la figure 4, on a porté en abscisse les hauteurs et en ordonnée les biomasses de chaque placette. On a superposé les hauteurs et les biomasses des modèles précédents (Bédéneau, 1988) et celles des ajustements obtenus ici. II apparaît que les premiers modèles sont valides jusqu'à des
Fig 3. Analyse factorielle des correspondances du tableau de contingence de classes de variables dendrométriques par région. Mise en «individu supplémentaire" de la Région parisienne. $A<18$ : âge < à 18 ans, $A<20$ : âge $<$ à 20 ans, $A<30$ : âge $<$ à 30 ans, $A>30$ : âge > à 30 ans, $\mathrm{B}<36$ : biomasse à l'hectare < 36 t, $\mathrm{B}<53$ : biomasse à l'hectare $<53 \mathrm{t}, \mathrm{B}<$ 79 : biomasse à l'hectare $<79 \mathrm{t}$, $\mathrm{B}>80$ : biomasse à l'hectare > $80 \mathrm{t}, \mathrm{H}<11$ : hauteur $<11 \mathrm{~m}$, $H<13: 11 \mathrm{~m}<$ hauteur $<13 \mathrm{~m}$, $H<15: 13 m<$ hauteur $<15 \mathrm{~m}$, $H>15$ : hauteur $>15 \mathrm{~m}, \mathrm{DB} 12$ : accroissement en biomasse < 12 t/ha, DB17 : 12 t/ha < accroissement en biomasse < 17 t/ha, DB27 : 17 t/ha < accroissement en biomasse < 27 tha, DB+t : accroissement en biomasse $>27$ tha, $\mathrm{DH} 10$ : accroissement en hauteur $<10$ $\mathrm{dm}(\mathrm{ou}=0), \mathrm{DH} 19: 10 \mathrm{dm}<$ accroissement en hauteur $<19$ dm, Dh34 : 19 dm < accroissement en hauteur < $34 \mathrm{dm}$, $\mathrm{DH}++$ : accroissement en hauteur $>34 \mathrm{dm}$.

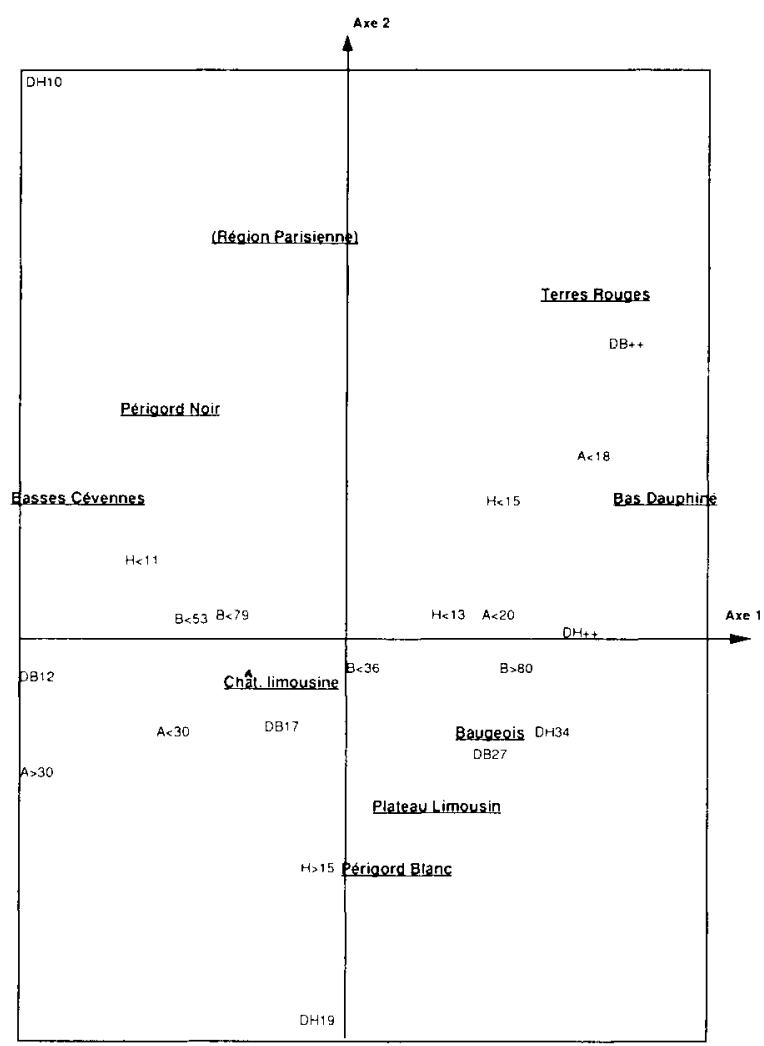




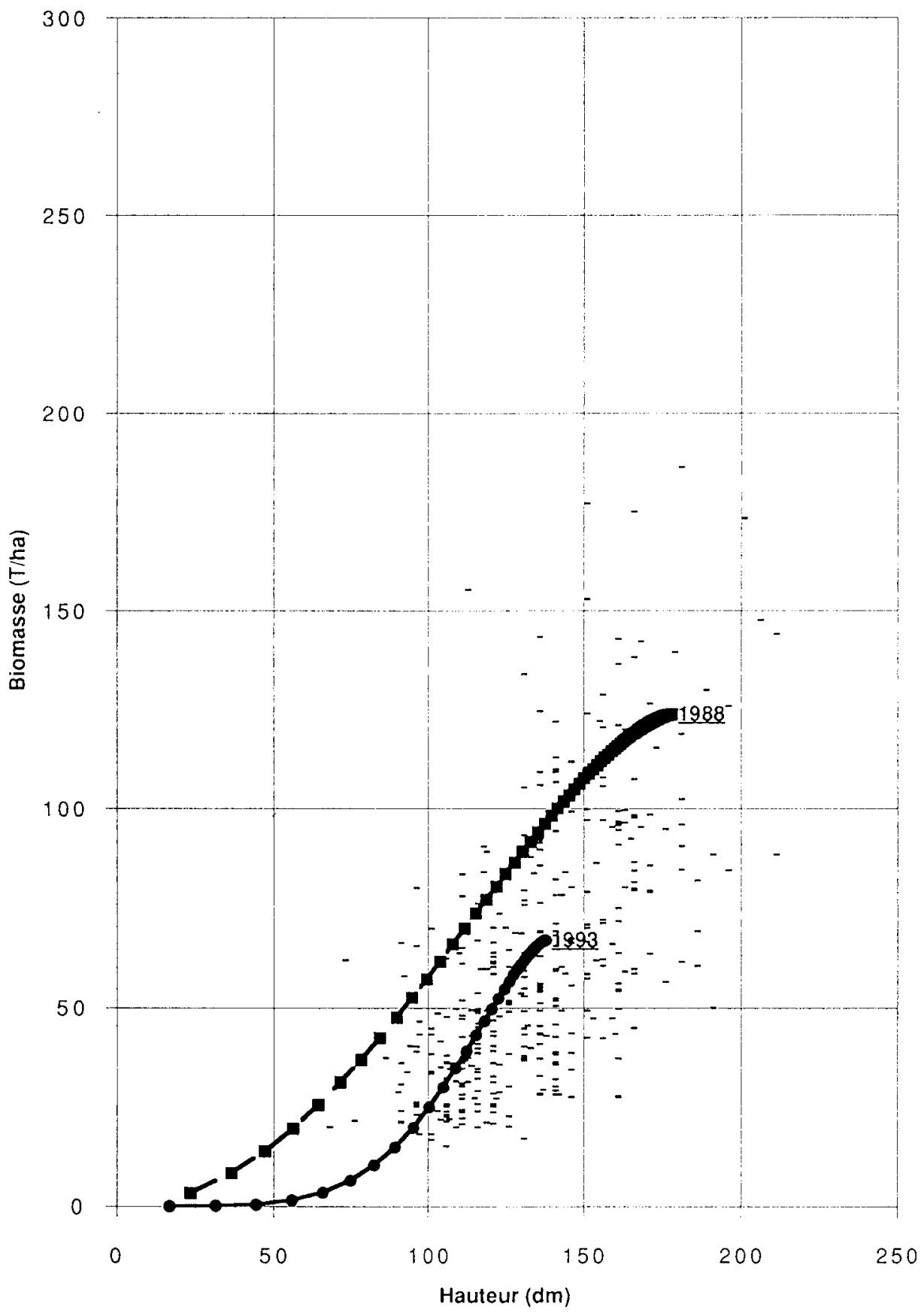

Fig 4. Relation hauteur-biomasse : modèles ajustés dans la présente étude et modèle précédent. 


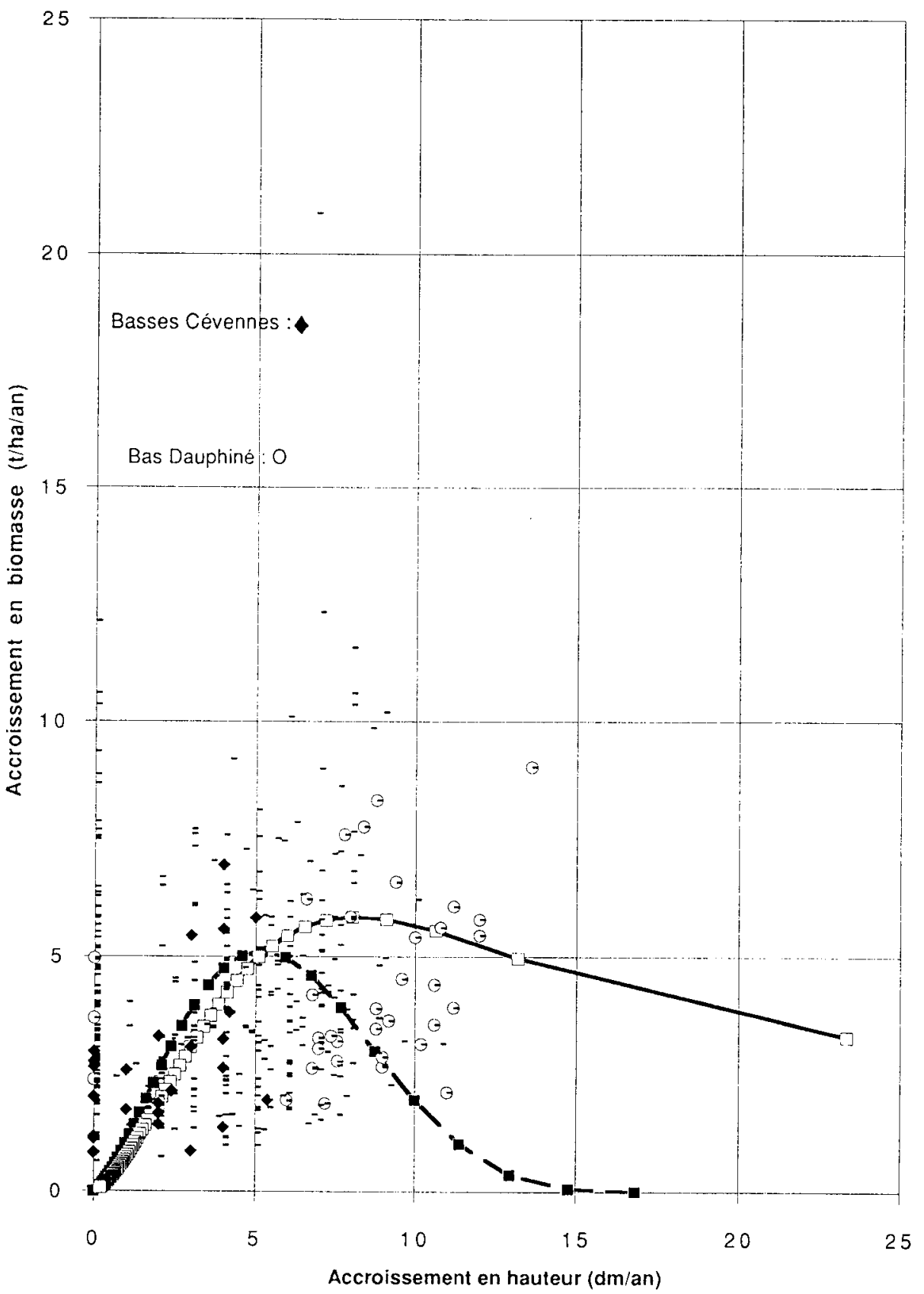

Fig 5. Relation hauteur-biomasse : accroissements courants par an et juxtaposition des modèles. 
hauteurs de $18 \mathrm{~m}$ et des biomasses à l'hectare de l'ordre de $120 \mathrm{t}$. Cependant la courbe passe au dessus des points les plus bas, et en revanche ne poursuit pas sur les points les plus élevés (d'une hauteur supérieure à $20 \mathrm{~m}$ ). Les modèles proposés ici rendent bien compte de la forme du nuage, mais culminent à des hauteurs de $14 \mathrm{~m}$ et des biomasse à l'hectare de $80 \mathrm{t}$.

Sur la figure 5 , on a porté les accroissements courants par an en hauteur et biomasse observés dans les points IFN et ceux déduits des modèles. On retrouve tous les points dont l'accroissement en hauteur est nul, puis les points rangés par accroissements courants. Les modèles partagent le nuage jusqu'à 0,6 $\mathrm{m}$ d'accroissement en hauteur et $5 \mathrm{t}$ de biomasse, mais après ils ne tiennent pas compte des valeurs plus élevées, ainsi que de la forme allongée du nuage. On peut constater qu'à une faible production de biomasse peuvent être associés ou bien de fort accroissements en hauter dans le jeune âge, ou bien au contraire de faibles accroissements dans les parcelles vieillissantes.

Pour illustrer les disparités entre régions on a porté, sur la figure 5 , une région de faible productivité (losanges), les BassesCévennes, et une région très productive (cercles), les Plateaux et collines du BasDauphiné. On constate qu'effectivement la performance de cette région est mieux expliquée par le modèle supérieur. En revanche, les Basses-Cévennes n'ont pas du tout la même croissance et sont plutôt sous le modèle ici ajusté. II existe cependant une grande variation à l'intérieur même de ces régions.

\section{DISCUSSION}

La surface du point de sondage IFN correspond à la surface minimale d'échantillonnage dans le cas particulier du taillis de châtaignier (Cabanettes, 1989). C'est pourquoi on a pu utiliser des données ini- tialement destinées à un inventaire à grande échelle, en remplacement de placettes temporaires, usuellement installées pour les études de production (Décourt, 1973).

Les résultats proposés ici diffèrent de ceux présentés dans l'étude réalisée précédemment (Bédéneau, 1988). La figure 4 met en évidence ces divergences : on constate que les ajustements obtenus avec les placettes fournies par les organismes de recherches et de développement se situent plus haut que ceux obtenus avec les données de l'IFN. Ceci paraît normal dans la mesure où les points IFN sont répartis de manière aléatoire dans les régions forestières, au pro rata de la réprésentativité de l'espèce, alors que les organismes de recherches installent des placettes en contrôlant le maximum de facteurs, c'està-dire dans des conditions édaphiques et des paramètres sylvicoles comparables, ces placettes ne sont pas forcément représentatives de la région considérée.

Deux résultats sont à souligner, d'abord le fait que la région forestière influe peu sur la croissance des peuplements : à l'intérieur d'une même région, on rencontre à la fois de très beaux peuplements et d'autres moins productifs. Ce résultat pourrait être expliqué par des variations locales de profondeur du sol, ou d'ensouchement, ou de pratiques culturales (rotations). Une nuance est à apporter pour certaines régions, telles les Basses-Cévennes, où les hauteurs se situent plutôt dans la partie basse de la courbe, ce qui avait été montré par De Champs (1972).

L'autre résultat est que la croissance d'un peuplement semble déterminée : les courbes de la figure 5 partagent le nuage de points des accroissements courants, et semblent indiquer qu'un passage d'une faible productivité à une plus élevée est bien improbable. Ceci peut signifier que la potentialité d'un peuplement, indépendante de la région de production (au sens IFN), suit une loi, prévisible par des variables dendrométriques. Ceci impliquerait que les 
facteurs de production ne dépendraient que de l'intervention humaine (recépage), pour les peuplements retenus ici : on peut envisager qu'à la suite des recépages successifs il y aurait un nombre limité de variations génétiques au sein de l'unité d'échantillonnage, et on tendrait donc vers une seule potentialité de croissance. $\mathrm{Ce}$ phénomène de diminution du potentiel génétique a déjà été montré sur le chêne vert traité en taillis par Yacine (1987). On peut également penser à des variations génétiques des provenances de châtaignier, comme suggéré par Frascaria et Lefranc (1992).

Cependant ces propos doivent, en outre, être tempérés par l'hypothèse faite que le nombre de brins était invariant en 5 ans. Ce qui est vrai pour des taillis de châtaignier à partir de 15 ans environ, mais inexact pour les taillis plus jeunes, dans lesquels il y a une forte mortalité des brins dominés. On ne peut pas reconstituer exactement le stock de biomasse ligneuse présent il y 5 ans : on la sous-estime.

Enfin il est vraisemblable qu'une part importante de la variation est due à un effet "station" : le même type de sol pouvant se retrouver dans des régions forestières différentes, à l'inverse des types de sols différents pouvant être présents dans une même région forestière.

On constate enfin que les régions en voie d'abandon, dans lesquelles l'exploitation traditionnelle du taillis a cessé, possèdent les peuplements les plus vieux et donc les moins productifs.

\section{CONCLUSION}

L'analyse des résultats fournis par cette étude aboutit à des chiffres de production moyenne plus faibles que ceux préalablement proposés : pour le modèle moyen d'évolution en biomasse sèche, la produc- tion courante maximale est de 4,3 t $^{-1} \mathrm{a}^{-1}$. $a^{-1}$ à 9 ans, la production moyenne maximale étant de 3 toha-1 $\bullet \mathrm{an}^{-1}$ à 12 ans. II en est de même pour les hauteurs : alors que, dans la précédente publication, le modèle avait une asymptote à $17,9 \mathrm{~m}$ et un allongement de $2,3 \mathrm{~m}$ la première année, l'asymptote est ici de $13,8 \mathrm{~m}$ et la croissance en hauteur de la première année de $1,7 \mathrm{~m}$. En revanche, la croissance en hauteur est encore trouvée maximale la première année. Le même modèle peut s'appliquer à l'ensemble des régions, qui sont plutôt discriminées par leur taux de croissance, lui-même fonction des interventions humaines.

Les peuplements les plus productifs, caractérisés par un nombre élevé de brins, ont une production courante maximale de 12 t•ha- ${ }^{-1} \cdot$ an $^{-1}$ à 9 ans et à 12 ans une production moyenne maximale de 8 t•ha ${ }^{-1}$. $\mathrm{an}^{-1}$. Ces chiffres sont inférieurs à ceux obtenus avec certaines espèces traitées en taillis à courtes rotations (TCR), en culture intensive, avec du matériel végétal très amélioré, tel le peuplier : rendement de $40 \mathrm{t}$ de matière sèche par hectare à 7 ans (soit environ une moyenne de 13 t• ha ${ }^{-1} \cdot \mathrm{an}^{-1}$ ). En revanche ces chiffres sont comparables à ceux escomptés dans les TCR de Séquoia en France ou obtenus avec l'eucalyptus, et le même mode de traitement, au Portugal, de l'ordre de 9 toha-1 an $^{-1}$ (Cannell, 1988 ; Auclair, 1986). Ces résultats montrent l'intérêt du châtaignier pour la production de biomasse ligneuse, car il croît naturellement en ne demandant des dépenses notables que lors de la coupe. Les TCR avec d'autres essences que le peuplier nécessitent des soins intensifs, coûteux, pour un rendement équivalent.

On voit aussi que la présence humaine est nécessaire au bon maintien de ces peuplements qui, non exploités, produisent peu.

Cette étude sera poursuivie par l'extrapolation de résultats de ce type à l'ensemble 
des régions forestières concernées, mais aussi, avec les mêmes données, par la conversion des tarifs de biomasse en tarif de volume. Outre la biomasse indifférenciée, à usage de trituration, le châtaignier fournit du bois de qualité, employé en menuiserie, parqueterie. La forme des arbres et les caractéristiques mécaniques du matériau prennent alors une importance capitale, et il est vraisemblable qu'à ce niveau d'étude la région forestière soit déterminante dans la qualité des produits. Enfin le châtaignier traité en taillis est souvent présent sous les futaies (Pin maritime en Périgord, Pin sylvestre en Sologne, etc) et il serait intéressant de mesurer l'impact des réserves sur le taillis, et réciproquement celui du taillis sur la futaie.

\section{REMERCIEMENTS}

Ce travail a nécessité l'intervention de plusieurs échelons de l'IFN, je remercie MM Bazire, Balleydier, Chevrou, Rousseau et Guillaume de leur aimable collaboration.

\section{RÉFÉRENCES}

ADDAD (1989) Manuel de référence, $250 p$

AFOCEL (1983) Production de biomasse : taillis à courte rotation. Assoc Forêt Cellul Nangis, $214 p$

Auclair D (1986) Productivité des espèces ligneuses forestières. Biomasse Actualités 9 , 26-34

Auclair D, Bigé MC (1984) Une méthode d'évaluation régionale de la biomasse des taillis à partir des données de l'Inventaire Forestier National. Application à la région Centre. Ann Sci For 41 (4) 405-426

Bédéneau $M$ (1988) Croissance du taillis de châtaignier en France : premiers résultats. Ann Sci For 45 (3) 265-274

Bouchon J (1974) Les tarifs de cubage, ENGREF, $57 p$

Bouvier A, Gélis F, Huet S, Messean A, Neveu P (1985) Manuel d'utilisation de CS-NL, INRA, laboratoire de Biométrie, Jouy-en-Josas, $180 \mathrm{p}$

Cabanettes A (1989) Une méthode pour l'estimation de la biomasse ligneuse aérienne dans les jeunes taillis, Acta Oecol Oecol Appl 10 (1) $65-80$

Cannell MGR (1982) World forest biomass and primary production data. Academic Press London, $391 \mathrm{p}$

Cannell MGR (1988) The scientific background. In: Biomass Forestry in Europe (FC Hummel, W Palz, G Grass, eds) Elsevier EEC, 83-137

Cost ND, McClure JP (1984) Biomass inventory and assessment in the Southern United States. Biomass 6 (182) 15-24

De Champs J (1972) La production des taillis de châtaignier. AFOCEL, rapport Annuel, 219278

Décourt N (1973) Protocole d'installation et de mesures des placettes de production semipermanentes. INRA-CNRF, Nancy $25 p$

Frascaria N, Lefranc M (1992) Le commerce de la châtaigne : un nouvel aspect dans l'étude de la différenciation génétique de population de châtaigniers (Castanea sativa Mill) en France. Ann Sci For 49 (1) 75-79

Guéro MC, Houllier F (1990) La base de données de l'Inventaire forestier national. Nature et utilisation de ses données dynamiques. Bull Rech Agron Gembloux 25 (1) 49-64

Inventaire forestier national (1985) But et méthodes de l'Inventaire forestier national. Ministère de l'Agriculture, direction des Forêts, $67 \mathrm{p}$

Lavoie G, Vallée G (1982) Inventaire des espèces et cultivars potentiellement valables pour la production de biomasse ligneuse. Rapport ENFOR LAU-X (56), $48 \mathrm{p}$

Osumi S (1983) Applicabilité de la fonction de Richards à l'analyse de croissance de l'arbre. In: Mesures des biomasses des biomasses et des accroissements forestiers, INRA Publ, Colloques de l'INRA, $N^{\circ} 19,77-86$

Ottorini JM, Nys C (1981) Application des données de l'Inventaire forestier national à l'étude de la production du pin sylvestre en Margeride. I. Étude de la croissance en hauteur. Ann Sci For 38 (2) 223-236

Paavilainen E (1981) Biomass yields and management of natural coppice stands. IEA Rep NE (19) $50 p$ 
Pagès $L$ (1986) Lois de croissance en biomasse du taillis. Le robinier dans le val de Loire. Ann Sci For 43 (4), 533-555

Pardé J (1980) Forest Biomass. For Abstr 41 (8) 343-362

Rullier-Bréval B (1985) Croissance d'un taillis de châtaignier après coupe. Thèse doct Univ Paris XI, $142 \mathrm{p}$

Sennerby-Forsse L, Mitchell CP (1987) Fuelwood production strategies. Swed Univ Agric Sci Uppsala, $155 \mathrm{p}$
Tomaselli I (1982) Liquefaction of wood. In: Energy from forest biomass (W Ramsay-Smith, ed) Academic Press, New York, 209-220

Tomassone R, Lesquoy E, Millier C (1983) La régression, nouveaux regards sur une ancienne méthode statistique. Masson, Paris, $180 \mathrm{p}$

Yacine A (1987) Une étude d'organisation de la diversité génétique inter et intra-population chez le chêne vert (Quercus ilex L.). Thèse 3 cycle, USTL Montpellier, $75 p$ 